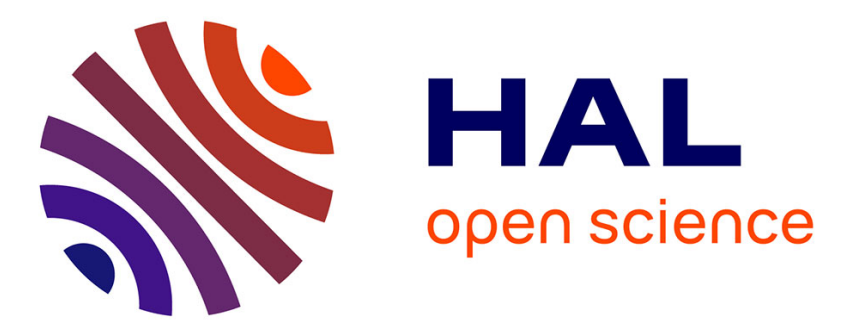

\title{
Traitements informatiques de la notation du jeu instrumental pour instrument de musique mécanique: la tonotechnie
}

\author{
Paola Sanchez, R. Hopp
}

\section{- To cite this version:}

Paola Sanchez, R. Hopp. Traitements informatiques de la notation du jeu instrumental pour instrument de musique mécanique: la tonotechnie. Journal de Physique IV Proceedings, 1994, 04 (C5), pp.C5-641-C5-644. 10.1051/jp4:19945138 . jpa-00252815

\section{HAL Id: jpa-00252815 https://hal.science/jpa-00252815}

Submitted on 1 Jan 1994

HAL is a multi-disciplinary open access archive for the deposit and dissemination of scientific research documents, whether they are published or not. The documents may come from teaching and research institutions in France or abroad, or from public or private research centers.
L'archive ouverte pluridisciplinaire HAL, est destinée au dépôt et à la diffusion de documents scientifiques de niveau recherche, publiés ou non, émanant des établissements d'enseignement et de recherche français ou étrangers, des laboratoires publics ou privés. 


\title{
Traitements informatiques de la notation du jeu instrumental pour instrument de musique mécanique : la tonotechnie
}

\author{
P. SANCHEZ et R. HOPP*
}

CNRS-LAS, Traverse du Siphon, 13012 Marseille, France

* Facteur d'Orgue, Château des Confines, 84300 Monteux, France

Résumé : "Tonotechnics" was the term which used to denote the art of driving embossed cylinders used for the manufacture of automated musical instruments.

"The art of the organ builder" written in the $18^{\text {th }}$ century by Dom Bedos de Celles describes the technique.

Nowadays the term is used more widely to include the transcription of musical notes not only on cylinders but on other materials such as paper or cardboard, and more particularly on the cards used for mechanical organ music.

A machine using "tonotecnics" designed by Robert Hopp has just been presented at the 1992 september music fair in Paris. The purpose of the machine is to produce perforated cards for mechanical organ music using a musical file (format MIDIFILE) recorded on an ordinary musical sequencer.

Until now perforating the cards was a long and often hazardous task frequently subject to error. Today, thanks to this machine one can not only reproduce the authentic manner of playing with greater ease and precision but also imagine a new, wider scope for the composition of mechanical organ music.

Some parts of the musical score composed by M. Michel PORTAL for the film " $l$ 'anniversaire du poisson lune" were only made possible by the design of this machine.

Its originality lies in its capacity to reproduce the traditional sounds of the instrument and thanks to sophisticated technology to improve the quality of the cards necessary to produce such music.

In the near future we hope to restore to perfect musical order mechanical organ music cards which are too old or badly damaged for use.

Currently prized by collectors but impossible to exploit these cards could be scanned by means of a camera or a coupled charge device in order to transcribe their original music onto new cards.

Thus the cultural wealth of mechanical organ music could be saved from oblivion.

\section{Introduction}

La Tonotechnie était autrefois l'art de piloter les cylindres à picots utilisés dans la fabrication d'instruments de musique automatisés. On trouve l'explication de cette technique dans un recueil đu XVIII ${ }^{e m e}$ siècle, écrit par Dom Bedos de Celles : "L'art du Facteur d'Orgue". Par extension cette technique s'applique de nos jours à la notation sur d'autres supports (carton, papier) et en particulier sur les cartons de l'orgue de Barbarie.

Une machine réalisée à partir de cette technique en collaboration avec Robert Hopp (inventeur du procédé) vient d'être présentée au Salon de la Musique à Paris en septembre 1992. Cette machine permet la création du carton perforé utilisé par l'orgue de Barbarie à partir d'un fichier musical au 
format MIDIFILE $\left({ }^{1}\right)$ enregistré depuis un séquenceur du commerce. Cette façon de procéder permet de respecter le jeu instrumental et ouvre des horizons nouveaux pour la création de nouvelles musiques. Le perçage des cartons était jusqu'à présent une opération très longue et sujette à beaucoup d'erreurs. La facilité de création et la précision du perçage obtenue à l'aide de l'ordinateur permettent d'envisager une augmentation rapide de la création musicale pour orgue de Barbarie.

\section{Le noteur}

Les noteurs sont les fabricants de ces cartons qui doivent en premier lieu arranger le morceau en fonction de la tessiture de l'instrument et ensuite réaliser le perçage du carton. Ce travail fastidieux et long (environ 10.000 perforations pour une seule musique) restreignait jusqu'à ce jour le répertoire. L'utilisation de l'informatique moderne permet maintenant de percer des cartons d'une façon beaucoup plus pratique et on assiste aujourd'hui à un événement merveilleux autant qu'étrange : la renaissance d'une invention ancienne, le carton perforé, qui utilisait déjà le principe informatique "oui ou non", grâce à sa descendance, l'Informatique Musicale.

La Notation est le travail qui consiste à noter la musique sur les supports de mémoire musicale (carton et papier perforé, cylindres picotés etc...) servant aux instruments de musique mécanique (orgues de Barbarie, Limonaires, Orchestrions, Pianolas) et, en l'occurrence, à faire des petits trous dans du carton qui passe ensuite dans les orgues de Barbarie. L'utilisation de l'ordinateur ne remplace pas le noteur, mais elle lui permet de se concentrer sur l'essentiel de son travail : la musique. Le côté fastidieux de la perforation est pris en charge par la machine. Ce travail était fait jusqu'à présent manuellement.

\section{Notation Assités par Ordinateur (NAO)}

Avec la notation assistée par ordinateur de Robert Hopp, les choses deviennent plus simples : le musicien fait un arrangement sur un séquenceur du commerce, soit en jouant directement sur un clavier de synthétiseur, soit en composant sur l'écran de l'ordinateur à l'aide d'un éditeur de partition. Il respecte les particularités de l'orgue, son étendue et ses possibilités sonores. Il sort sa musique sous forme de fichier "MIDIFILE" (standard très utilisé en informatique musicale) et on la présente à la machine à perforer à commande numérique. On indique le standard du carton désiré, sa largeur, l'emplacement des notes sur les pistes, la division ainsi que d'autres paramètres techniques. En quelques minutes, le carton est percé au centième de millimètre près avec un contrôle rigoureux des notes.

\section{Description technique}

La station est composée d'un ordinateur relié à une interface de puissance, reliée elle-même à une machine à perforer à commande numérique. Les arrangements sont faits à l'aide d'un séquenceur interne ou externe, auquel cas l'importation se fait à l'aide de disquettes de n'importe quel format et formatage. Tous les standards courants peuvent être récupérés : Atari, Mac, PC, etc..., pourvu que les fichiers aient été sauvegardés sous forme de "MIDIFILE". Le système transforme les fichiers

1 Le format MIDIFILE (Musical Instrument Digital Interface) est un standard de communication par l'intermédiaire de fichiers informatiques des musiques enregistrées à l'aide de séquenceurs musicaux. 
"MIDI( $\left.{ }^{2}\right)^{\prime}$ en une forme exploitable par le robot qui perce les cartons ou papiers. Les musiques sont introduites dans l'ordinateur par l'intermédiaire d'une interface MIDI. Cette interface reçoit d'un clavier musical les informations de hauteur et de durée des notes. Seules ces informations sont indispensables pour réaliser un carton perforé.

Un code MIDI représente la définition d'une liste de codes numériques que l'on compose en "message" représentant des gestes instrumentaux élémentaires. La liaison MIDI permet de connaitre un grand nombre de paramètres supplémentaires tels la vélocité d'enfoncement ou de relachement d'une note, le vibrato, etc... Le principe de fonctionnement de l'Orgue de Barbarie ne permet pas de traduire sur le carton tous les paramètres du jeu instrumental. Une partie du programme consiste à filtrer les notes et leur durée.

Il existe une grande variété d'orgues de barbarie dont les tessitures sont différentes. Pour s'adapter à la quasi-totalité des instruments, on utilise une table de conversion dont l'entrée est donnée par le code MIDI de la touche enfoncée sur le clavier. La sortie représente la position ou le rang du trou à percer par rapport à un bord du carton.

La difficulté pour le créateur de musiques nouvelles consiste à jouer sur un clavier en ignorant les notes ne faisant pas partie de la gamme. Bien évidemment, il y a souvent des erreurs et l'ordinateur se charge de les signaler. On peut également envisager une introduction de la partition à l'aide d'un éditeur de partition du commerce, mais on perd l'intérét de l'interprétation. Cette interprétation était auparavant due au noteur ; elle devient maintenant celle de l'interprète.

Dans un premier temps, pour des raisons de précision dans la mécanique d'entraînement du carton, nous avions décidé de réaliser un entrainement sans possibilité de recul. Pour gagner du temps sur le perçage, le bras qui porte le poinçon avait un mouvement bidirectionnel. Mais nous nous sommes aperçu rapidement de l'impossibilité de réaliser certain cas de figure. Donc il était obligatoire de pouvoir faire reculer le carton. Cette manoeuvre a été facilitée par la grande précision des moteurs pas à pas d'entrainement et par le système de guidage du carton. Actuellement, il est possible de percer quatre cartons simultanément à la vitessse de dix mètres par heure. Tous les paramètres de la machine sont fournis au programme par le noteur : taille du carton, longueur du poinçon, vitesse de défilement, écartement entre les touches, etc...

Les indications de hauteur et de durée sont insuffisantes dans certain cas. Pour des orgues utilisant des instruments mécaniques, il faut tenir compte du temps de latence pour la mise en route des instruments à percussion par exemple. Il faut donc ajouter un temps en début et en fin des notes pour synchroniser l'ensemble de la composition.

Des problèmes nouveaux sont apparus, la fragilité du carton dans le cas d'accords très long. Nous avons donc introduit un paramètre permettant de décider de la création de ponts à l'intérieur du perçage pour assurer la solidité du carton. La vitesse de défilement normale du carton est de 60 millimètres par seconde. La note la plus courte possible est donnée par la largeur du poinçon (3,5 ou $4 \mathrm{~mm}$ en général). Dans un système de 4 temps, dans un tempo d'une mesure par seconde, la triple croche est impossible à percer. La machine à perforer traitera la triple croche avec la valeur la plus petite possible pour l'orgue : une valeur de poinçon. Une rafale de triple croche sera traitée par la machine comme une seule longue note, une triple croche isolée sera traitée avec la valeur approchée du coup de poinçon. Il y a cependant l'échappatoire de la vitesse de défilement : rien n'interdit de noter plus lentement et de tourner ensuite la manivelle plus vite. La machine nta pas besoin de savoir quel système on utilise, elle travaille en temps réel.

2 Codage d'évènements musicaux permettant l'interconnexion de machines numériques dédiées à la musique. 
Le système permet de corriger note par note le temps de réponse des instruments. Ce qui veut dire que les perforations tiennent compte des systèmes de lecture, de transmission, bref des particularités de chaque type d'orgue. La résolution du système est de l'ordre du centième de millimètre ce qui correspond à 0,0001666 seconde. En perforation manuelle, une personne expérimentée perforera avec une précision d'un demi millimètre, done avec une précision 50 fois inférieure. Le système peut perforer des cartons jusqu'à 500 millimètres de large et un nombre de touches illimité.

\section{Conclusion}

Les avantages du système sont nombreux.

La liberté musicale : les musiciens peuvent se concentrer sur leur vocation première au lieu de se perdre dans les petits trous et de se décourager avec des machines à perforer manuelles trop compliquées.

La précision : le système perce exactement ce que le musicien entend, au dix millième de seconde près. La plus grande précision anciennement atteinte est cinquante fois inférieure.

La rapidité : Auparavant, il fallait attendre des semaines et des mois pour obtenir les cartons. Il en était de même pour les fabrications spéciales qui était presque impossibles. Le système permet actuellement des traitements en quelques heures.

La polyvalence musicale : les arrangements sont désormais possibles dans toutes les tonalités, en accompagnement avec ou sans ligne mélodique, orchestrés selon la nécessité de l'utilisation. Le même titre peut exister en plusieurs versions différentes.

La liberté de composition : les musiciens et arrangeurs peuvent s'exprimer sur l'orgue de Barbarie sans les contraintes pratiques de la perforation. La facilité d'adaptation de répertoires nouveaux : en un clin d'oeil il est possible de faire des musiques d'origines diverses. Chaque pays a ses musiques folkloriques propres. "La Java Bleue" est très connue des Champs-Elysées à la Canebière, mais à Tokyo et Djakarta, ou même à Zurich, il faut composer des musiques plus adaptées aux répertoires nationaux.

Mais pour que cela fonctionne, il faut tout de même respecter certaines règles, étudier la gamme de l'instrument et la respecter, suivre les instructions sur les gammes en ce qui concerne les canaux MIDI et les octaves, ne faire que les arrangements et interprétations musicalement et techniquement possibles sur un orgue de Barbarie. Pas de notes trop courtes, pas d'accords avec plus de notes simultanées que la soufflerie de l'orgue peut en supporter.

Cependant tous les styles de musique sont possibles. On peut s'exprimer en free jazz, en musique contemporaine, ou en bourrée auvergnate ... En principe, la machine va perforer exactement ce que l'on entend sur l'ordinateur, mais le son (le timbre) de l'orgue étant différent, il faudra un tout petit peu d'imagination pour le résultat final.

Apparemment connu, l'orgue de Barbarie offre de nombreuses possibilités en fait ignorées. A l'origine, instrument de musique populaire, facile d'emploi car il suffit de tourner une manivelle, il est en train de devenir un instrument de musique à part entière. Certains compositeurs ont suivi l'exemple de Mozart, Haydn, Beethoven et recommencent à écrire pour la boîte magique : Marius Constant, Martial Solal, Pierre Charial mais également Michel Portal qui a écrit une musique pour le film "l'anniversaire du poisson lune" dont certaines séquences pour orgue de Barbarie ont été réalisées par cette machine.

L'originalité de cette réalisation tient également dans le mariage entre le respect de l'instrument traditionnel et l'amélioration du support musical par une technologie avancée. 